\title{
Hard-hearted CRP
}

$T_{h}$ he data will be what the data will be - regardless of a community's fondest wishes. And the latest data from the most comprehensive epidemiological study carried out so far on C-reactive protein (CRP) and its predictive value for coronary heart disease (CHD) in the general population indicate that CRP is not as strong a predictor of heart disease among healthy individuals as has been touted in the press (Danesh et al., ref. 1).

"Nobody would have been happier than I had it been a powerful marker," said Mark Pepys, of the Royal Free and University College Medical School, London, and coauthor of the Danesh et al. paper, to the JCI. "Our study is not trying to knock CRP. We're just trying to get the right answer."

Pepys has been working on CRP for 30 years and was an author of one of the first detailed studies of CRP in patients with CHD (2), and of the key first study of CRP in acute coronary syndromes (3) that triggered the current avalanche of CRP work in relation to CHD risk. The story from the initial publications on CRP, through its overwhelming hype, to the publication of Danesh et al., appears to be the usual cautionary tale of the rise and fall of a superstar. The Danesh et al. report comes on the heels of a 2003 publication recommending that clinicians offer optional CRP measurements to individuals whose 10 -year risk of CHD is $10-20 \%$, but also emphasized that further large-scale epidemiological studies were required (4).

"Recommendations about routine use of CRP testing for CHD risk may have been premature," Pepys said, "and influenced by the enormous amount of publicity surrounding CRP." Indeed, reports of President Bush's CRP levels made all the papers.

Following the 2002 publication of a very large epidemiological study by Ridker et al. indicating that CRP was a better CHD predictor than cholesterol (5), many of the subsequent reports, both in the lay press and in clinical reviews, that mentioned that study did not make clear the potential weaknesses of the study from a clinical perspective, and they generally ignored the key behavioral features of CRP.

CRP is a sensitive blood protein marker of inflammation, infection, and tissue

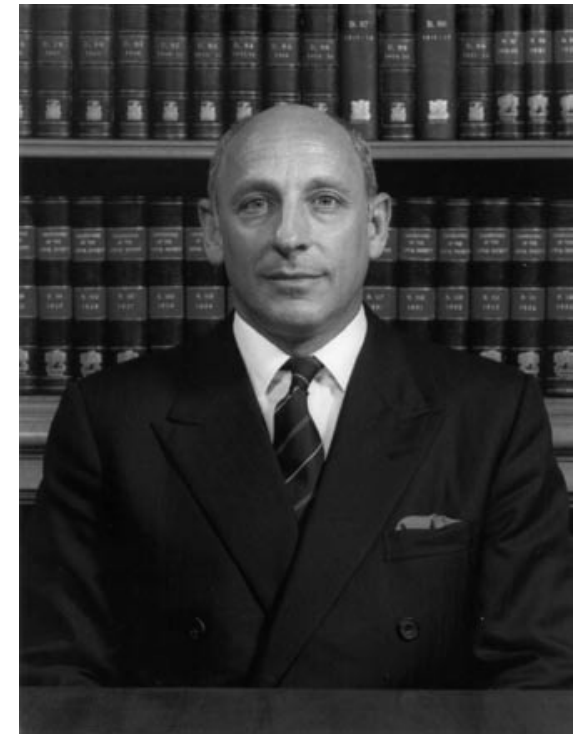

Work by Mark Pepys and colleagues indicates that the claims for CRP may be overdone.

damage. "It is completely non-specific. It goes up when you're sick for almost any reason," Pepys explained. "To describe it as a specific marker of cardiovascular disease is very misleading. You need to know everything clinically that is going on with an individual to place [CRP concentration levels] in perspective."

Although the first research studies on CRP indicated it was a strong predictor of CHD, this conclusion was always accompanied by many caveats. In fact, an editorial by Lori Mosca (6) accompanying

\section{"The important thing is, what extra value do you get from a CRP measurement? And the answer is, relatively little."}

the Ridker et al. paper raised numerous questions and noted that while the study held promise, it was far from complete from a clinical perspective. Regarding the very high statistical significance reported in Ridker et al., Mosca noted that "statistical significance can be inflated with large sample sizes, of course, whereas the clinical importance of a difference may be minimal. This fact should be taken into consideration as statistics are translated into clinical strategy."

In his discussion with the JCI, Pepys made a similar point concerning epidemiological studies in general: "People are impressed by studies with huge numbers of participants, but if there are only a small number of events [incidents of disease], it is essential to be cautious, especially when dealing with relatively modest risk factors."

The Ridker et al. study, although one of the largest (totaling 27,939 women), had 371 CHD events. Further, it divided the population into quintiles of CRP concentrations, again reducing the number of events per group. Having a relatively low number of events that are further subdivided into many categories can possibly cause sampling errors. The Danesh et al. study had 2,459 CHD events, more than six times the total in Ridker et al., and the average follow-up time on the population was 19 years as compared to 8 years in Ridker et al.

Numerous smaller studies have also indicated that increased CRP levels are associated with CHD risk. Danesh et al. includes meta-analyses of these studies. "The total number of events in the meta-analysis [of the 11 major studies published since 2000] is about the same as this one in our study," Pepys told the JCI. "And the relative risks from the meta-analysis and this study are the same." They both end up with a relative risk of 1.5 for the top third of CRP distribution compared to the bottom third, in contrast to 2- to 4-fold risk (depending on the type of cardiovascular event and whether hormone replacement therapy is taken into account) in Ridker et al.

A meta-analysis of all studies up to 2000 , comprising a total of about 1,900 events, indicated a relative risk of 1.9, higher than that of the later studies (7). Pepys suggested that this may reflect a recognized phenomenon in epidemiology, with "early publication bias favoring positive papers. Then, as more and more papers come out, we see what is called 'regression to the mean' and [in many cases] the association disappears. Danesh calls this 'regression to the truth."'

Still, with top third baseline CRP levels providing a 1.5 -fold relative risk of 
CHD, the question is whether this is still worth testing. "Statistically," Pepys said, "this is very significant. The question is how clinically significant is it. [Our work indicates] it is much less clinically significant than the established risk factors, such as being a smoker or [having a high] cholesterol level. So, the important thing is, what extra value do you get [from a CRP measurement]? And the answer is, relatively little.”

In addition, many researchers and clinicians note that for a test to be of use to the general population, several points should be met, including whether the substance being tested is directly implicated in the disease and whether there are interventions to reduce or alter the tested substance's effect or amount.

Pepys agrees, noting, for example, that "there are things that can be done when you see someone smoking or [who has] high cholesterol. But there is no evidence that lowering baseline CRP will affect someone's health and as yet no treatments that selectively and specifically reduce or inhibit CRP."

So, how disheartened should we be about CRP not being the Delphic oracle of heart disease? "Our study only questions the usefulness of routine CRP measurements [for CHD prediction] for people in the general population,” Pepys said. There are three quite different clinical contexts, he said, in which to look at CRP levels with regard to CHD: (a) in the general healthy population; (b) in people with acute coronary syndromes; and (c) in people who have just had a heart attack.

The data on the latter two, "where CRP values are dramatically higher than the very low baseline levels of the general healthy population, seem to be solid," Pepys notes. In these cases, "CRP is a good prognostic marker. The higher the CRP, the worse off the patient is likely to be. It is an incredibly useful test when you form an opinion about how sick a patient is."

It is CRP's relationship to illness in general that still holds appeal, and in Europe, CRP measurement is a widely used standard test. "It's the biochemical equivalent of taking a patient's temperature," Pepys said - it is a non-specific marker of simply being sick. But unlike temperature, CRP levels are not affected by things such as external temperature changes or taking an aspirin or other drugs, or indeed by any intervention that does not control the underlying disease process.

"CRP values correlate very well with how sick you think the patient is," Pepys explained. "If you do things that make the patient better, CRP falls; if you do things that are not helping, the CRP remains high."

In the past, US doctors, however, have not been as keen to utilize CRP in such a general way, preferring to rely on more specific markers for the patient's disease. So the use of this exquisitely nonspecific index of disease as a specific test for CHD risk has been rather surprising. And perhaps it is now due for reassessment.

\section{Laurie Goodman}

1. Danesh, J., et al. 2004. C-reactive protein and other circulating markers of inflammation in the prediction of coronary heart disease. N. Engl. J. Med. 350:1387-1397.

2. de Beer, F.C., et al. 1982. Measurement of serum $\mathrm{C}$-reactive protein concentration in myocardial ischaemia and infarction. Br. Heart J. 47:239-243.

3. Liuzzo, G., et al. 1994. The prognostic value of Creactive protein and serum amyloid A protein in severe unstable angina. N. Engl.J. Med. 331:417-424.

4. Pearson, T.A., et al. 2003. Markers of inflammation and cardiovascular disease: application to clinical and public health practice: a statement for healthcare professionals from the Centers for Disease Control and Prevention and the American Heart Association. Circulation. 107:499-511.

5. Ridker, P.M., et al. 2002. Comparison of C-reactive protein and low-density lipoprotein cholesterol levels in the prediction of first cardiovascular events. N. Engl. J. Med. 347:1557-1565.

6. Mosca, L. 2002. C-reactive protein - to screen or not to screen? N. Engl.J. Med. 347:1615-1617.

7. Danesh, J., et al. 2000. Low grade inflammation and coronary heart disease: prospective study and updated meta-analyses. BMJ. 321:199-204. 